\title{
OPEN Evaluating the effect of rice (Oryza sativa L.: SRNC05053-6-2) crude extract on psoriasis using in vitro and in vivo models
}

\author{
Sumate Ampawong ${ }^{1 凶}$, Kanchana Kengkoom ${ }^{2}$, Passanesh Sukphopetch ${ }^{3}$, \\ Pornanong Aramwit ${ }^{4,5}$, Watcharamat Muangkaew ${ }^{3}$, Tapanee Kanjanapruthipong ${ }^{1}$ \& \\ Theerapong Buaban ${ }^{2}$
}

Psoriasis is mainly caused because of inappropriate immune responses in the epidermis. Rice (Oryza sativa L.: SRNC05053-6-2) consists of anthocyanin, which exhibits strong antioxidative and antiinflammatory properties. This study aimed to evaluate the role of this black-coloured rice crude extract in alleviating the symptoms of psoriasis using human psoriatic artificial skin and an imiquimod-induced rat psoriasis model. Psoriasis-related genes, cytokines and chemokines were examined; in addition, the antioxidative and anti-inflammatory properties and the immunohistopathological features of this condition were studied. The results showed that the rice extract reduced the severity of psoriasis by (1) decreasing the epidermal thickness, acanthosis, hyperkeratosis, epidermal inflammation and degree of apoptosis induction via caspase-3, (2) increasing the expression levels of anti-inflammatory cytokines (IL-10 and TGF- $\beta$ ), (3) reducing the levels of pro-inflammatory cytokines (IL-6, IL-8, IL-20, IL-22 and TNF- $\alpha$ ), chemokines (CCL-20) and anti-microbial peptides (psoriasin and $\beta$-defensin), (4) enhancing the antioxidative property (Nrf-2), (5) downregulating the levels of psoriasis-associated genes (psoriasin, b-defensin, koebnerisin 15L and koebnerisin 15S) and (6) upregulating the levels of psoriasis-improving genes (caspase-14, involucrin and filaggrin). Thus, the extract appears to exert therapeutic effects on psoriasis through its antioxidative and immunomodulatory properties.

Psoriasis, a noncontagious chronic skin condition characterised by the excessively rapid proliferation of keratinocytes, is a common T cell-mediated inflammatory skin disease; it is associated with autoimmune responses that mainly attack the target cells in the skin, particularly keratinocytes and melanocytes, leading to cellular hyperplasia and post-inflammatory hyperpigmentation, respectively ${ }^{1-5}$. Psoriasis is considered an incurable, long-term and inflammatory skin condition with periods of improvement and worsening. Furthermore, it is one of the most common skin conditions closely related to mental illness, particularly in patients who present with generalised lesions ${ }^{6}$. The pathogenesis of psoriasis mainly involves the imbalance of epidermal immune responses, which is divided into four stages: initialisation, innate immune response, adaptive immune response and amplification loop $^{7}$. The initiation step is triggered by environmental and genetic factors (e.g. $L C E 3 B / L C E 3 C 1$ ) and leads to epidermal alterations such an upregulation in the expression level of $\beta$-defensin, which results in the formation of self-DNA/RNA and LL37 complexes and the activation of interferon (INF)- $\gamma$. Subsequently, dendritic cells are stimulated into the maturation stage and play a pivotal role in mediating the innate immune response by secreting several cytokines, such as transforming growth factor (TGF)- $\beta$, tumour necrotic factor (TNF)- $\alpha$ and interleukin (IL)-6. Consequently, these cytokines initiate T cell-mediated adaptive immune responses through Th-17, Th-1 and Th-22 systems and lead to the increased production of IL-17A, IL-17F, IL-22, IL-21, TNF- $\alpha$ and

\footnotetext{
${ }^{1}$ Department of Tropical Pathology, Faculty of Tropical Medicine, Mahidol University, Ratchawithi Road, Ratchathewi, Bangkok 10400, Thailand. ${ }^{2}$ Academic Service Division, National Laboratory Animal Center, Mahidol University, 999, Salaya, Puttamonthon, Nakorn Pathom 73170, Thailand. ${ }^{3}$ Department of Microbiology and Immunology, Faculty of Tropical Medicine, Mahidol University, Ratchawithi Road, Ratchathewi, Bangkok 10400, Thailand. ${ }^{4}$ Department of Pharmacy Practice, Faculty of Pharmaceutical Sciences and Center of Excellence in Bioactive Resources for Innovative Clinical Applications, Chulalongkorn University, PhayaThai Road, Phatumwan, Bangkok 10330, Thailand. ${ }^{5}$ The Academy of Science, The Royal Society of Thailand, Dusit, Bangkok 10330, Thailand. ${ }^{\circledR}$ email: am_sumate@hotmail.com
} 


\begin{tabular}{|l|l|l|}
\hline Component & Property & Test method \\
\hline Anthocyanin & $974.258 \mu \mathrm{g} / 50 \mathrm{mg} / \mathrm{ml}$ & HPLC \\
\hline Vitamin E & $1.06 \mathrm{mg}$ a-tocopherol/100 $\mathrm{g}$ & In-house methods of Nutrition Institute, Mahidol University, Thailand \\
\hline Vitamin D2 & $<0.05 \mu \mathrm{g} / 100 \mathrm{~g}$ & AOAC $(2016) 995.05$ \\
\hline Ascorbic acid & $3.35 \mathrm{mg} / 100 \mathrm{~g}$ & In-house methods of Nutrition Institute, Mahidol University, Thailand \\
\hline \multirow{2}{*}{ Antioxidative activity } & $34,544.00 \mu \mathrm{mole} \mathrm{TE} / 100 \mathrm{~g}$ & FRAP \\
\cline { 2 - 3 } & $2,537.63 \mathrm{mgAA} / 100 \mathrm{~g}$ & DPPH \\
\hline
\end{tabular}

Table 1. Composition of Nil Surin rice (Oryza sativa L.: SRNC05053-6-2) extract.

INF- $\gamma$. Therefore, both Th-1 and Th-17 cytokines induce keratinocytes to produce IL-20, which increases the proliferation of the cells during the auto-amplification step. Moreover, IL-1 $\beta$, IL- 6 and TNF- $\alpha$ are produced by keratinocytes and contribute to the activation of dendritic cells, thereby promoting and increasing the severity of the epidermal inflammation.

The severity of psoriasis mainly depends on the degree of immune response in the epidermis. Hence, substances that reduce the degree of epidermal inflammation might serve as candidates for alleviating psoriasis in the skin. Researchers claimed that pigmented-rice comprises anthocyanin, which demonstrates strong antioxidative, anti-microbial and immunomodulatory properties ${ }^{8,9}$. The black-coloured rice Oryza sativa L.: SRNC05053-6-2 or Mali-Nil-Surin rice exhibits a dark purple pericarp and consists of high levels of anthocyanin, vitamins B-1 and -2 , vitamin E and omega-3, -6 and -9; it is developed by several cooperation units in Thailand, particularly the (1) Surin Rice Research Center, (2) Sakon Nakhon Rice Research Center, (3) Division of Rice Research and Development, Rice Department, (4) Faculty of Technology and (5) Faculty of Science, Mahasarakham University, (6) Faculty of Medicine, Khon Kaen University and (7) School of Food Technology, Suranaree University ${ }^{10}$. Therefore, owing to its immunomodulatory and antioxidative properties, the crude extract of $O$ sativa L.: SRNC05053-6-2 might demonstrate some beneficial effects on psoriasis of the skin. The present study aimed to evaluate the efficacy of $O$ sativa L.: SRNC05053-6-2 extract in treating psoriasis using in vitro (full-thickness three-dimensional reconstituted human skin psoriasis) and in vivo (imiquimod-induced rat psoriasis) models. Several approaches, such as histopathology, immunohistochemistry, electron microscopy, Bio-Plex Multiplex immunoassay and quantitative reverse transcription-polymerase chain reaction (qRT-PCR), were used to determine the improvement criteria, which were based on the pathology and the levels of the cytokines, chemokines and genes related to psoriasis.

\section{Results}

A component of $O$ sativa L.: SRNC05053-6-2 extraction. High-performance liquid chromatography (HPLC) indicated a high concentration of anthocyanin in the rice extract (Table 1), in addition to both vitamin $\mathrm{E}$ and $\mathrm{C}$; however, vitamin $\mathrm{D}_{2}$ (ergocalciferol) was not detected in the extract. Furthermore, the extract comprised antioxidative properties when examined using the FRAP and DPPH antioxidative capacity assays.

Epidermal thickness and histopathological changes in psoriatic models after treatment with the extract. After treatment with the extract, the artificial psoriatic in vitro model demonstrated a significant decrease in epidermal thickness in all the treatment groups [extraction, betamethasone and calcitriol (D3)], when compared with the non-treated and intact skin specimens (Fig. 1A; i-v, xi). Similarly, a significant reduction in epidermal thickness was observed in all treatment groups in the imiquimod-induced psoriatic rat model when compared with the non-treated skin (Fig. 1A; vi-x, xii). Prior to the treatment, the imiquimodinduced psoriatic rats exhibited skin lesions such as redness, scaling and thickening (Fig. 1B). The psoriatic area and severity scores were rapidly increased 2 days post-induction and remained high on day 4 until the first day of treatment (Fig. 1B; xiv). The main epidermal histopathological changes observed were acanthosis with an increase in the number of rete ridges, dermatitis, folliculitis and hyperkeratosis (Fig. 1B; ix-xiii). Histopathological scores revealed that all treatment groups exhibited a significantly lower score than those in the non-treated rats (Fig. 1B; xv).

Antiapoptotic, antioxidative and anti-inflammatory effects of the extract on the psoriatic skins. Immunohistochemical staining of caspase-3, Nrf-2, IL-10, TGF- $\beta$, $\beta$-defensin and CCL-20 were performed to evaluate the antiapoptotic, antioxidative and anti-inflammatory properties of the extract on the psoriatic skin models with or without treatment. The results demonstrated that caspase-3 expression levels in all the treatment groups were significantly lower than those in non-treated skin, in both the in vitro and in vivo psoriatic models (Fig. 2A). However, the expression level of caspase- 3 in the artificial psoriatic skin specimen, which received standard treatment, was significantly lower than that observed in the specimens that were treated with the extract. The antioxidative capacity, observed by determining the Nrf-2 expression level, was significantly higher in the groups treated with the extract, when compared with those in the other treatment groups in the artificial and rat psoriatic models (Fig. 2B).

Compared with the non-treated skin and standard treatment groups, IL-10 expression was found to be significantly increased in artificial skin treated with the extract; likewise, the expression levels of IL-10 in the rat psoriatic skins treated with $2.5 \%$ of the extract and calcitriol were significantly higher than those in the other 
A
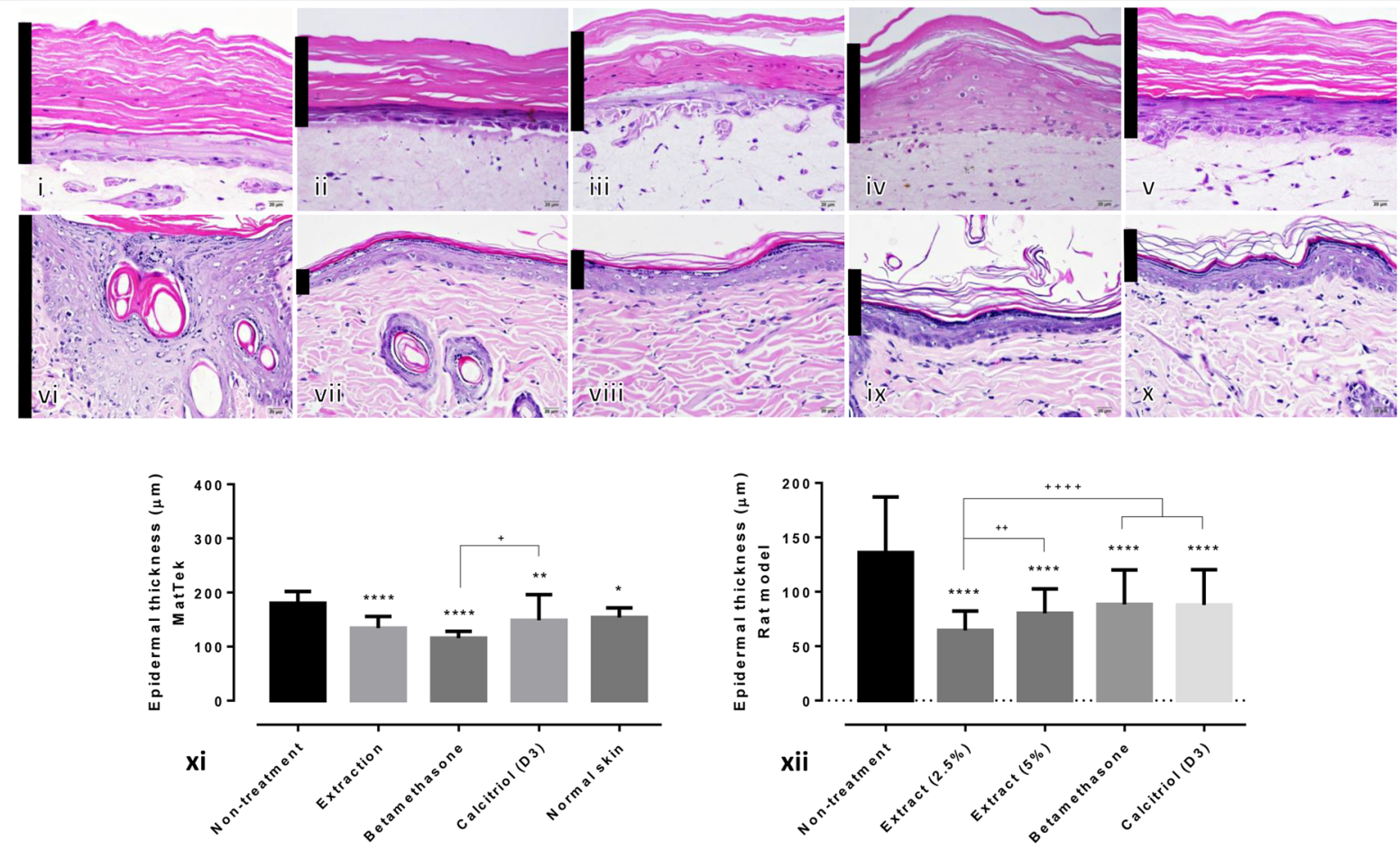

B
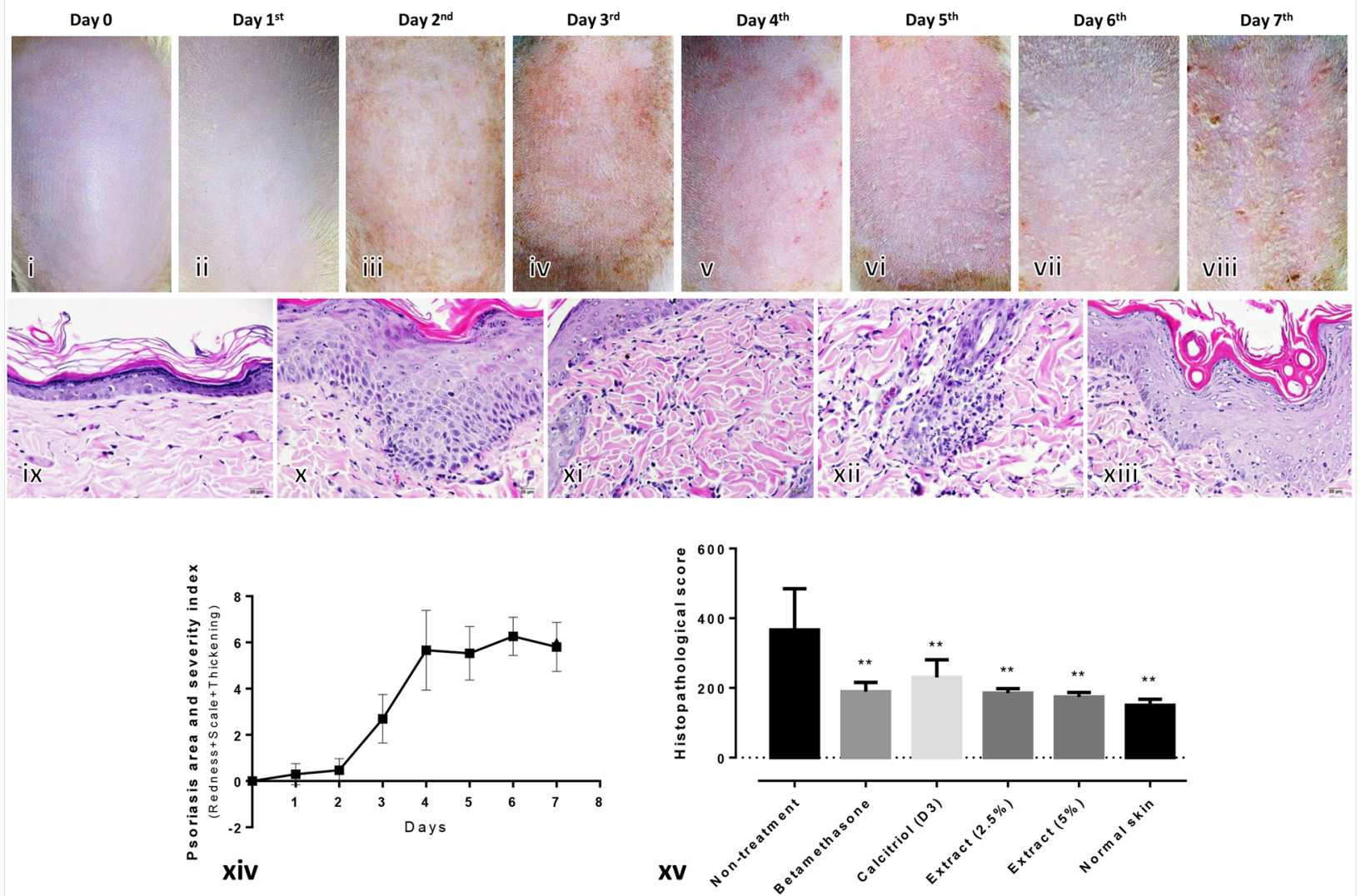

Figure 1. Histopathological evaluation of specimens from artificial psoriatic human skin and imiquimod-induced psoriatic rat skin: (A) Epidermal thickness at day 7 post-treatment in the artificial psoriatic (i-v) and rat psoriatic (xi, $x$ ) skins stained with hematoxylin and eosin (magnification, $\times 400$ ). (i, vi) non-treatment; (ii, vii) extraction; (iii, viii) betamethasone; (iv, ix) calcitriol (D3); (v, x) normal skin groups. Bar graphs comparing the epidermal thickness in the artificial psoriatic (xi) and rat psoriatic (xii) skins. (B) Anatomical and histopathological appearances in imiquimod-induced psoriasis rats; gross skin lesion after induction with $62.5 \mathrm{mg}$ of imiquimod from day 0 to day 7 (i-viii). Histopathological changes in rat psoriatic skin after 7 days of induction (ix-xiii). Ix, normal epidermis; $x$, acanthosis; xi, dermatitis; xii, folliculitis; xiii, hyperkeratosis. (xiv, xv) Line graph comparing the psoriatic area and severity index from day 0 to day 7 post-induction in each group (xiv). Bar graph comparing the histopathological scores among each treatment group (xv). 
A
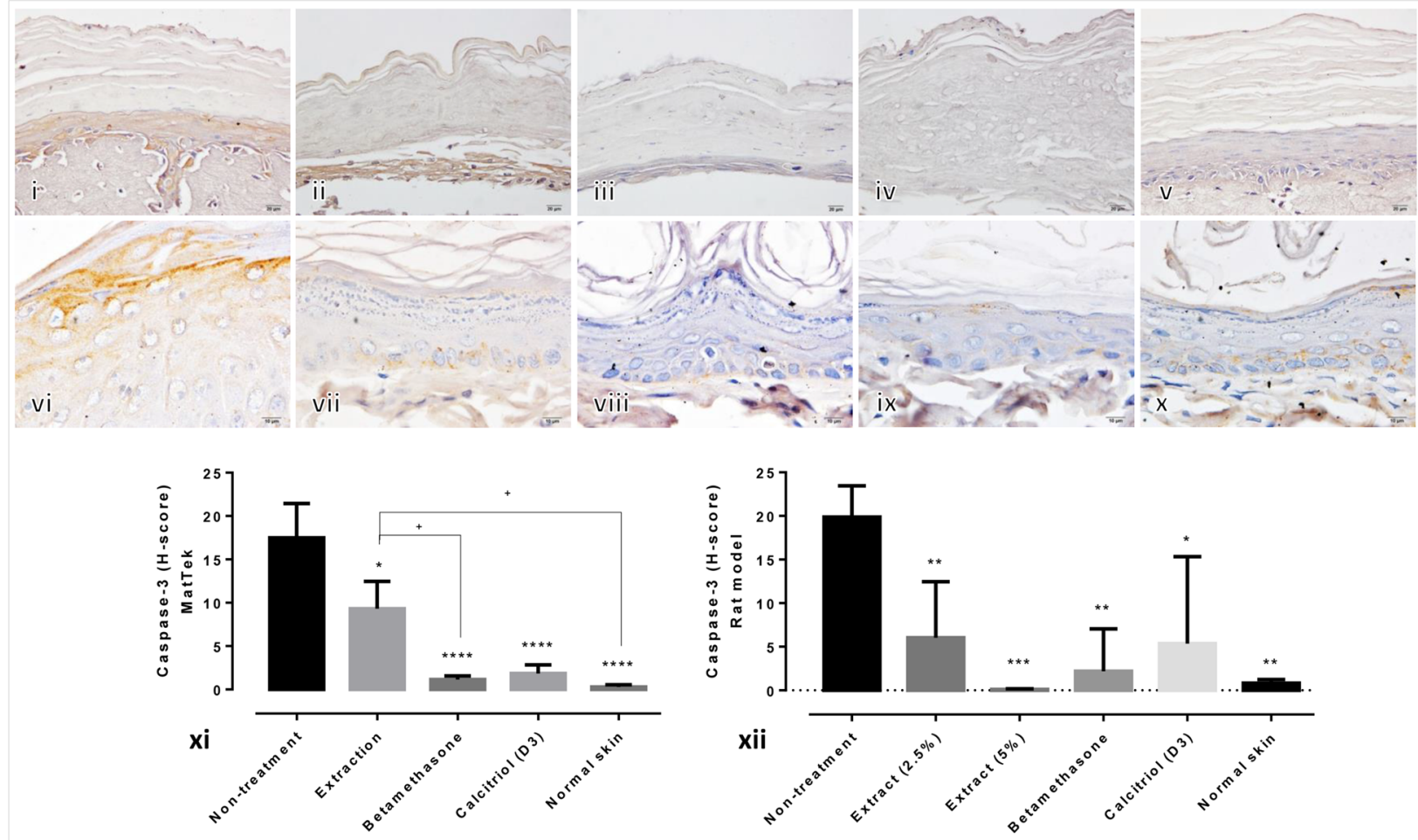

B
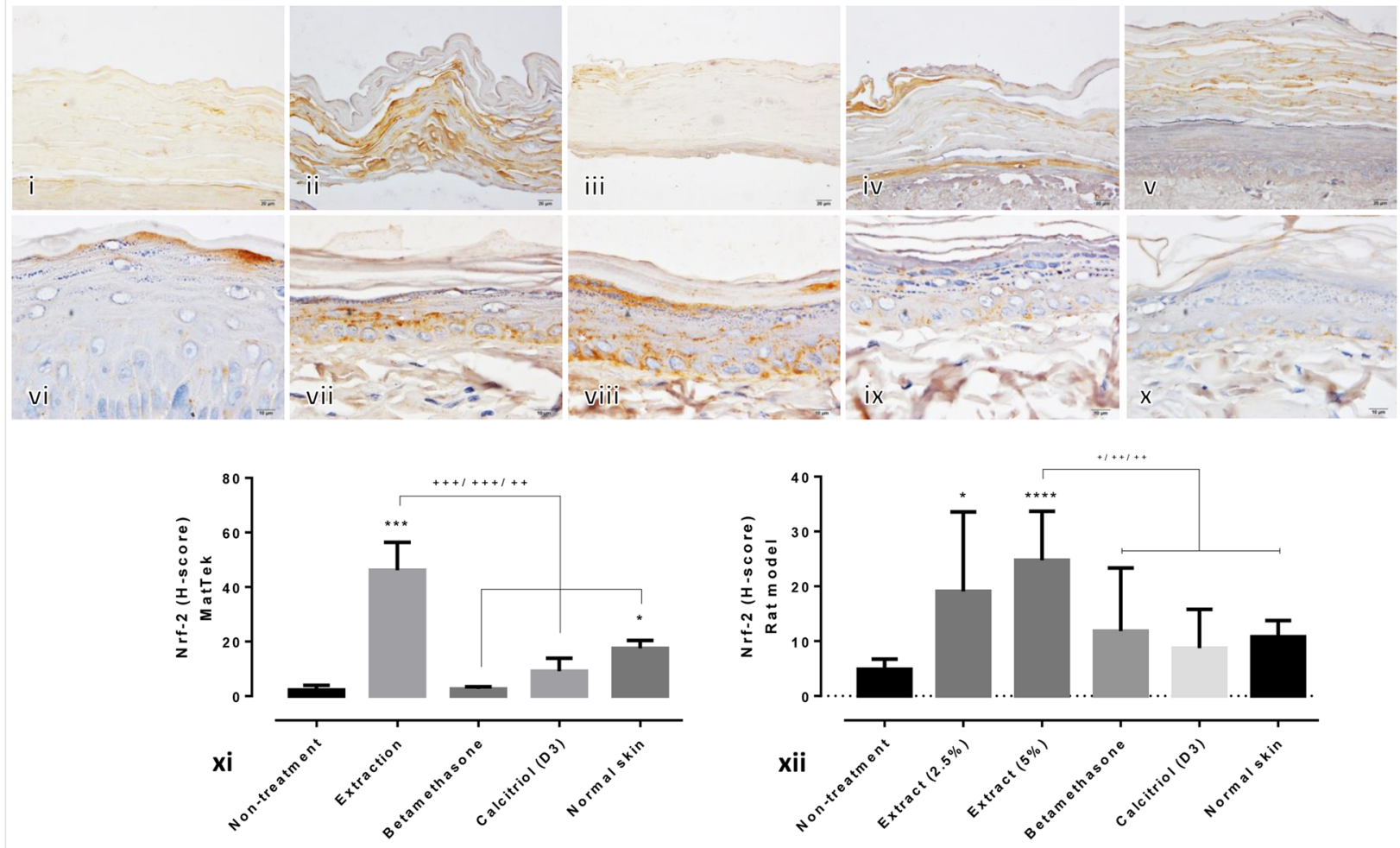

Figure 2. Epidermal apoptotic and antioxidative levels in artificial psoriatic human and imiquimod-induced psoriatic rat skins: (A) Caspase- 3 immunolabeling of the epidermis at day 7 post-treatment in the artificial psoriatic $(\mathrm{i}-\mathrm{v})$ and rat psoriatic $(\mathrm{vi}-\mathrm{x})$ skins (magnification, $\times 400)$. (i, vi) non-treatment; (ii, vii) extraction; (iii, viii) betamethasone; (iv, ix) calcitriol (D3); and (v, x) normal skin. (xi, xii) Comparison of epidermal caspase-3 expression levels in artificial psoriatic (xi) and rat psoriatic (xii) skins. (B) Nrf-2 immunolabeling of the epidermis at day 7 post-treatment in the artificial psoriatic (i-v) and rat psoriatic (vi-x) skins (magnification, $\times 400$ ). i and vi, non-treatment; (ii, vii) extraction; (iii, viii) betamethasone; (iv, ix) calcitriol (D3); and ( $\mathrm{v}, \mathrm{x}$ ) normal skin. (xi, xii) Comparison of Nrf-2 epidermal expression in the artificial psoriatic (xi) and rat psoriatic (xii) skins. 
A
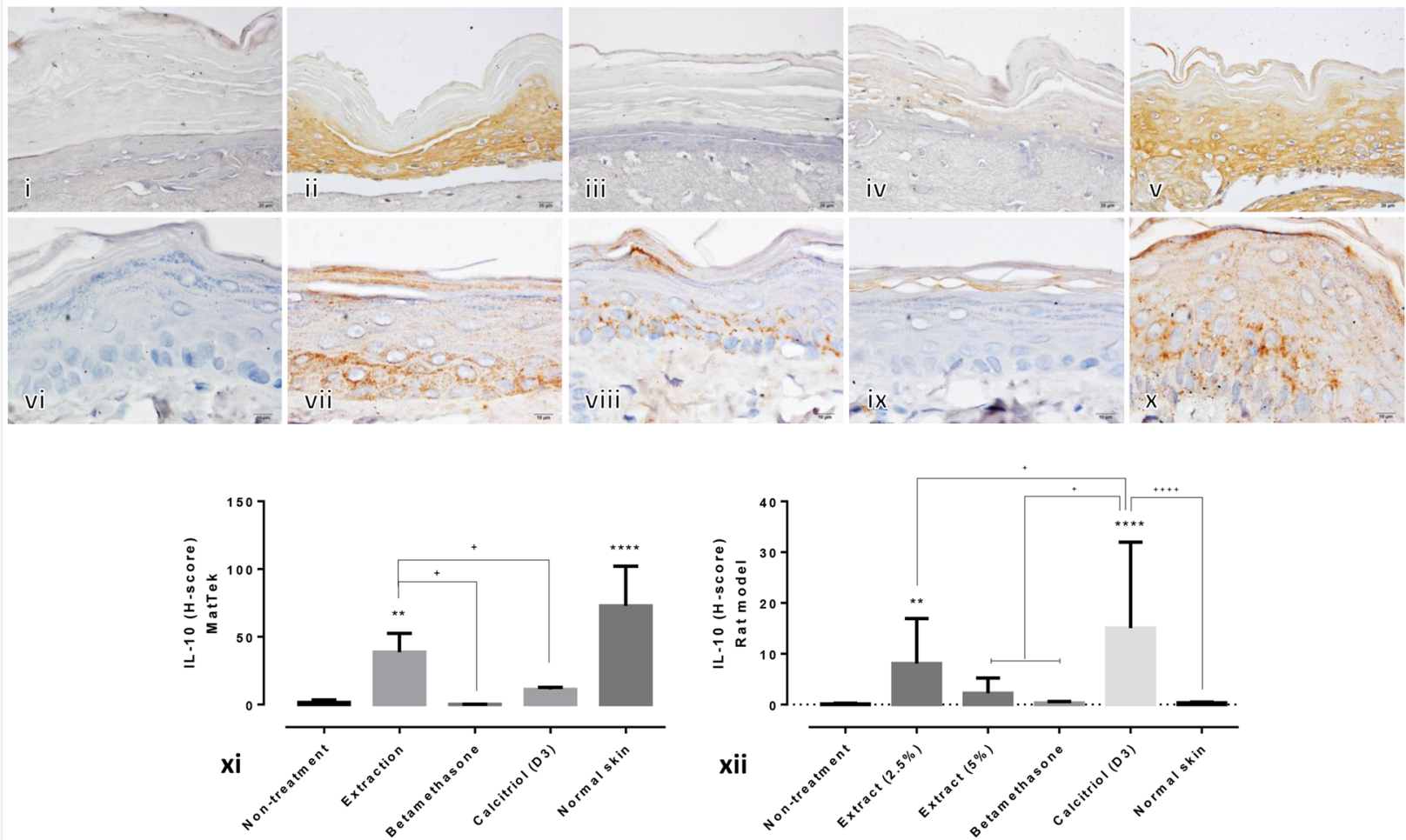

B
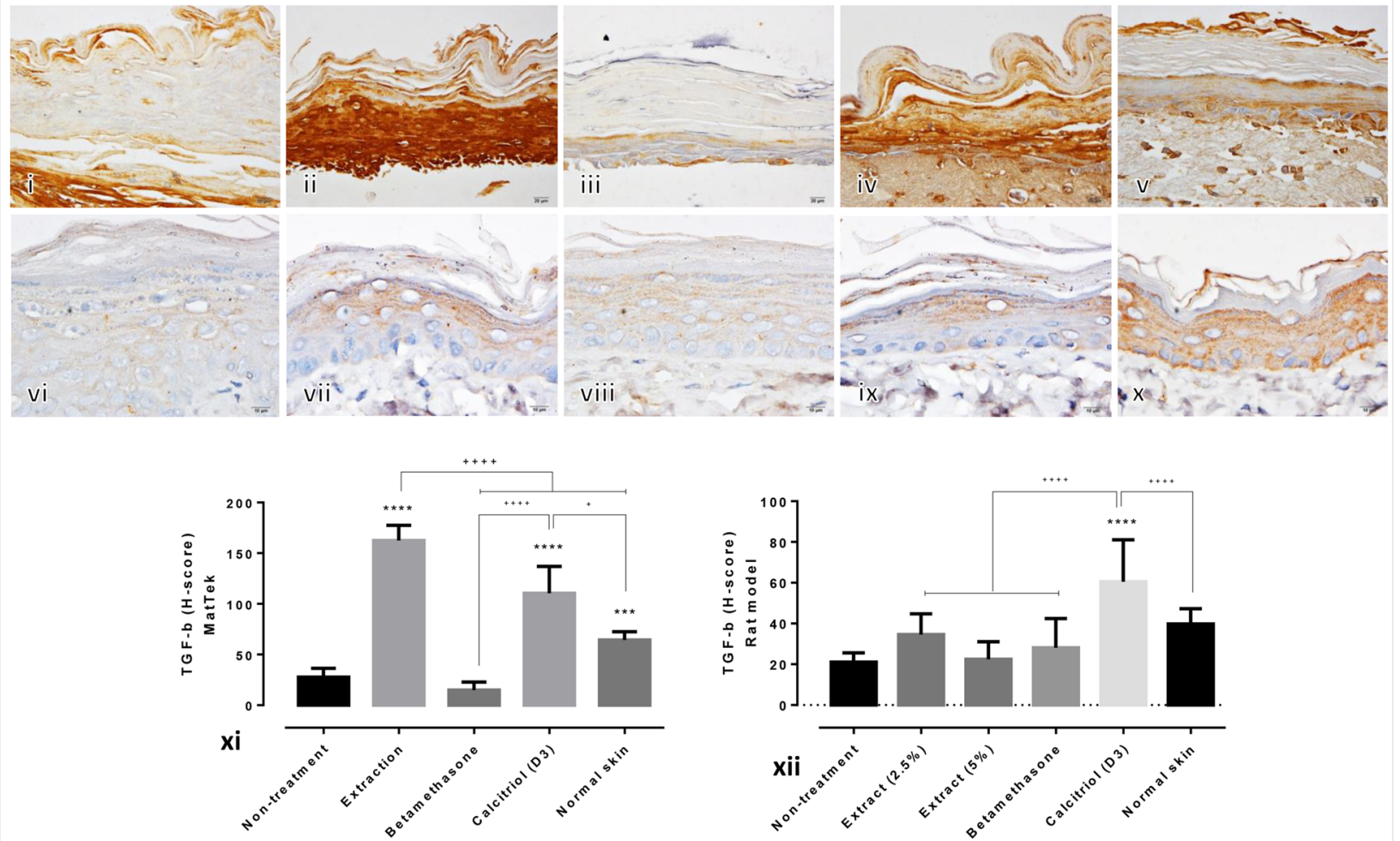

Figure 3. Epidermal anti-inflammatory cytokine levels in artificial psoriatic human and imiquimod-induced psoriasis rat skin specimens: (A) IL-10 immunolabeling of the epidermis at day 7 post-treatment in the artificial psoriatic (i-v) and rat psoriatic (vi-x) skins (magnification, $\times 400$ ). (i, vi) non-treatment; (ii, vii) extraction; (iii, viii) betamethasone; (iv, ix), calcitriol (D3); and (v, x), normal skin. (xi, xii) Bar graph comparing the expression levels of epidermal IL-10 among the various groups in the artificial psoriatic (xi) and rat psoriatic (xii) skin specimens. (B) TGF- $\beta$ immunolabeling of the epidermis at day 7 post-treatment in the artificial psoriatic $(\mathrm{i}-\mathrm{v})$ and rat psoriatic $(\mathrm{vi}-\mathrm{x})$ skins (magnification, $\times 400$ ). (i, vi) non-treatment; (ii, vii) extraction; (iii, viii) betamethasone; (iv, ix), calcitriol (D3); and (v, x) normal skin. (xi, xii) Bar graph comparing the expression levels of epidermal TGF- $\beta$ among the various groups in the artificial psoriatic (xi) and rat psoriatic (xii) skins. 
groups (Fig. 3A). The expression levels of TGF- $\beta$ in the artificial skin specimens treated with the rice extract and calcitriol were significantly higher than those in the other groups (Fig. 3B; i-v, xi). Moreover, only calcitrioltreated rat psoriatic skin demonstrated significantly upregulated levels of TGF- $\beta$ compared with the other groups (Fig. 3B; vi-x, xii).

In addition, $\beta$-defensin expression levels were significantly decreased in rat psoriatic skin specimens treated with the extract (2.5\%) and calcitriol, and the CCL-20 expression level was significantly downregulated in the $2.5 \%$ extract-treated rats, compared with those in the other treatment groups (Fig. 4A). The betamethasonetreated rats showed higher expression levels of CCL-20 than any of the other treatment groups. Spearman's correlation test revealed that the expression level of epidermal caspase- 3 was positively correlated with the epidermal thickness and acanthosis scores; alternatively, a negative correlation was observed between TGF- $\beta$ and the epidermal thickness (Fig. 4B). Furthermore, the epidermal thickness was positively correlated to the acanthosis and histological scores. Additionally, positive correlations were observed between the (1) histological and inflammation scores, (2) histological and acanthosis scores and (3) acanthosis and inflammatory scores.

Effect of rice extract on the expression levels of related psoriatic genes in artificial skin. The mRNA expression levels of caspase-14, filaggrin, involucrin, psoriasin and koebnerisin 15L/S were examined in the artificial skin specimens treated with or without the rice extract, calcitriol and dexamethasone using qRTPCR. The results demonstrated that epidermal caspase-14 was upregulated in skin specimens treated with the extract and calcitriol compared with that in the non-treatment group (Fig. 5A). Furthermore, the expression level of caspase-14 in specimens treated with extract was significantly higher than that in the calcitriol-treated specimens. When compared with that in the non-treated group, the mRNA levels of filaggrin were significantly higher in all the treatment groups; in addition, a significant difference was observed between the extract-treated and dexamethasone-treated specimens. The expression level of epidermal involucrin was higher in the extractand calcitriol-treated specimens than in the non-treated skin specimens and the extract group presented with the highest expression level among all groups. Psoriasin was significantly downregulated in all the treatment groups compared with the non-treated skin specimens. Likewise, the expression level of koebnerisin was decreased following all treatments, except for the 15L-subclass in calcitriol-treated skin specimens. Significant correlations between the expression levels of these genes and some of the immunohistochemical markers (caspase-3, Nrf-2, IL-10 and TGF- $\beta$ ) were observed (Table 2). High expression levels of caspase-14 were related with the upregulation of Nrf-2 and involucrin and downregulation of psoriasin. The expression level of involucrin was positively correlated with those of Nrf-2, IL-10 and TGF- $\beta$, and it was negatively correlated with those of koebnerisin and psoriasin. Negative correlations between the expression levels of koebnerisin and Nrf-2, IL-10 and TGF- $\beta$ were noted. The decreased expression levels of caspase- 3 and Nrf- 2 were associated with the increase in the expression levels of filaggrin and psoriasin.

Cytokine production in artificial skin following treatment with the extract. Some of the important pro- and anti-inflammatory cytokines and chemokines were selected to evaluate the differences in their expression levels using the Bio-Plex Multiplex immunoassay. TNF- $\alpha$, IL-1 $\beta$, IL-2, IL-4, IL-6, IL-8 and IL-22 levels were significantly decreased after treatment with the rice extract compared with those in the non-treated skin specimens (Fig. 5B; i, iv-viii, xiii). The expression levels of TNF- $\alpha$ in the 400 and $800 \mu \mathrm{g} / \mathrm{ml}$ extract-treated specimens were significantly lower than those in the $200 \mu \mathrm{g} / \mathrm{ml}$ extract-treated specimens and those that received standard treatments. IL-1 $\beta$, IL-2, IL-4, IL-6 and IL-8 levels were significantly lower than those in the specimens that received standard treatment. In addition, the expression level of IL-22 was the lowest following treatment with $400 \mu \mathrm{g} / \mathrm{ml}$ of the extract compared with those in the other groups. Conversely, INF- $\gamma$, IL-1 $\alpha$, IL-10, IL-12, IL-17 and IL-21 levels were not different between the rice extract-treated and -untreated specimens. However, the specimens treated with betamethasone demonstrated significantly higher levels of TNF- $\alpha$, IFN- $\gamma$, IL- $1 \alpha$, IL-1 $\beta$, IL-2, IL-4, IL-6, IL-8, IL-10, IL-12, IL-17, IL-21 and IL-22 than the other specimens.

Antimelanogenicity and immunomodulatory capacities of the rice extract. Electron microscopy was used to examine the antimelanogenic and immunomodulatory capacities of the extract on PEG-induced allergic melanocytes. Gold immunolocalisations of IL-4, TGF- $\beta$, microphthalmia-associated transcription factor (MITF) and tyrosinase were found in the cytoplasm and nucleus of the melanocytes and mature and immature melanosomes (Fig. 6A). Scanning electron micrographs indicated that several melanosomes were produced in allergic melanocytes that were not treated with the extract compared with those in the extract-treated cells (Fig. 6B, C). MITF immunoreactivity was observed in the transmission electron micrographs in both immature and mature melanosomes (Fig. 6D, E). The expression levels of IL- 4 and TGF- $\beta$ in melanocytes treated with 200 and $800 \mu \mathrm{g} / \mathrm{ml}$ of rice extract were significantly higher than those in the non-treated cells, particularly in the nucleus and cytoplasm (Fig. 6F, G). The levels of MITF and tyrosinase in immature melanocytes treated with 200 and $800 \mu \mathrm{g} / \mathrm{ml}$ of the rice extract were lower than those in the non-treated cells (Fig. $6 \mathrm{H}, \mathrm{I}$ ). In addition, MITF and tyrosinase levels were downregulated in melanocytes treated with $800 \mu \mathrm{g} / \mathrm{ml}$ of the extract compared with those in melanocytes treated with $200 \mu \mathrm{g} / \mathrm{ml}$ of the extract and the non-treated cells.

\section{Discussion}

Several natural products, such as curcumin ${ }^{11,12}$, cannabinoid ${ }^{13}$, anthocyanin (and its derivatives), were developed for the alleviation of psoriasis ${ }^{14-19}$. These products exert the desired effects on psoriasis via different mechanisms. Curcumin extract, when applied both topically and orally, acts as an antiseptic and decreases inflammation by downregulating the levels of inflammatory cytokines such as TNF- $\alpha$, IFN- $\gamma$, IL-2, IL-17A, IL-17F, IL-22 and IL-23, thereby reducing both parakeratosis and hyperkeratosis in the psoriatic lesions ${ }^{11,12}$. Moreover, curcumin is 
A
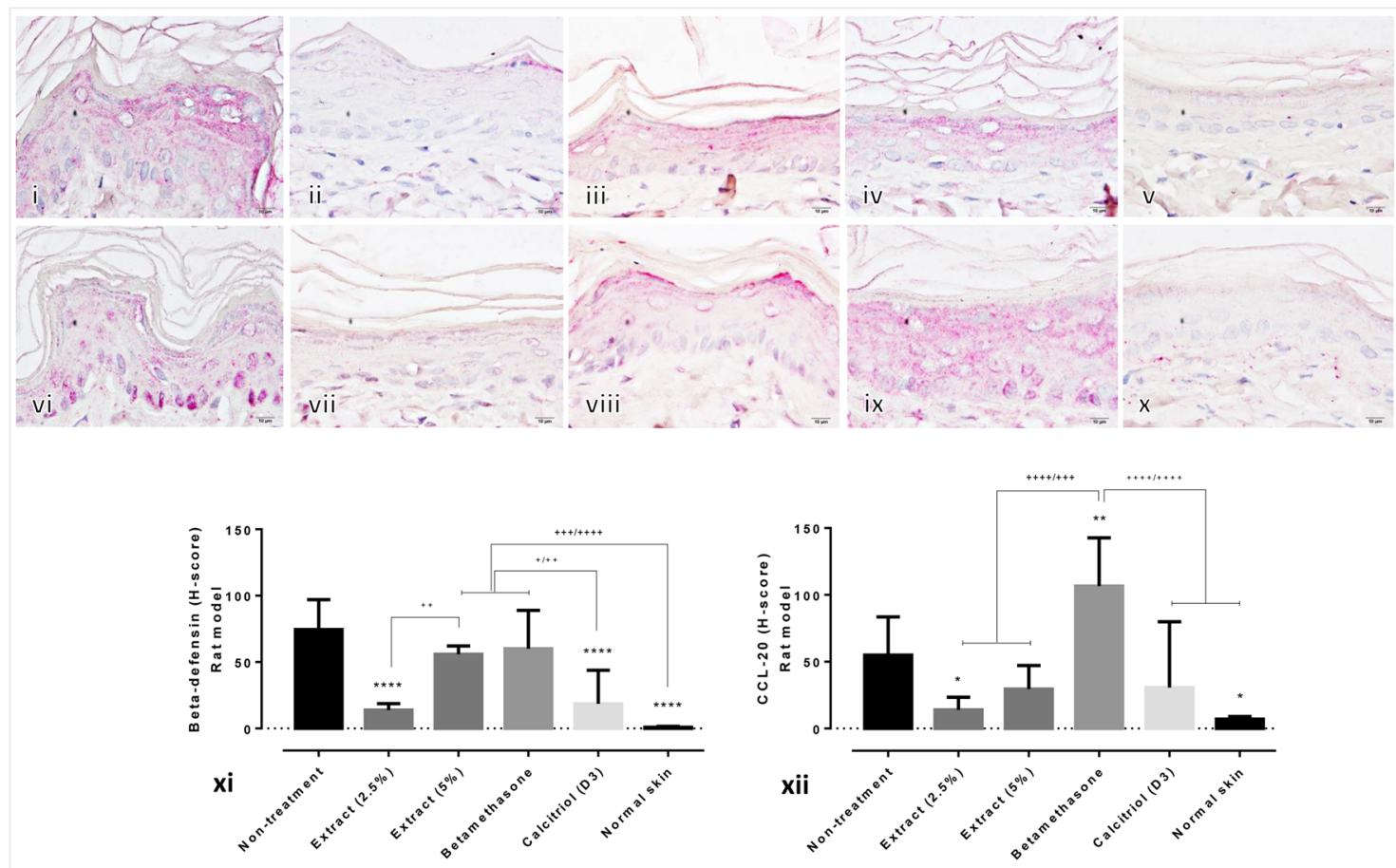

B
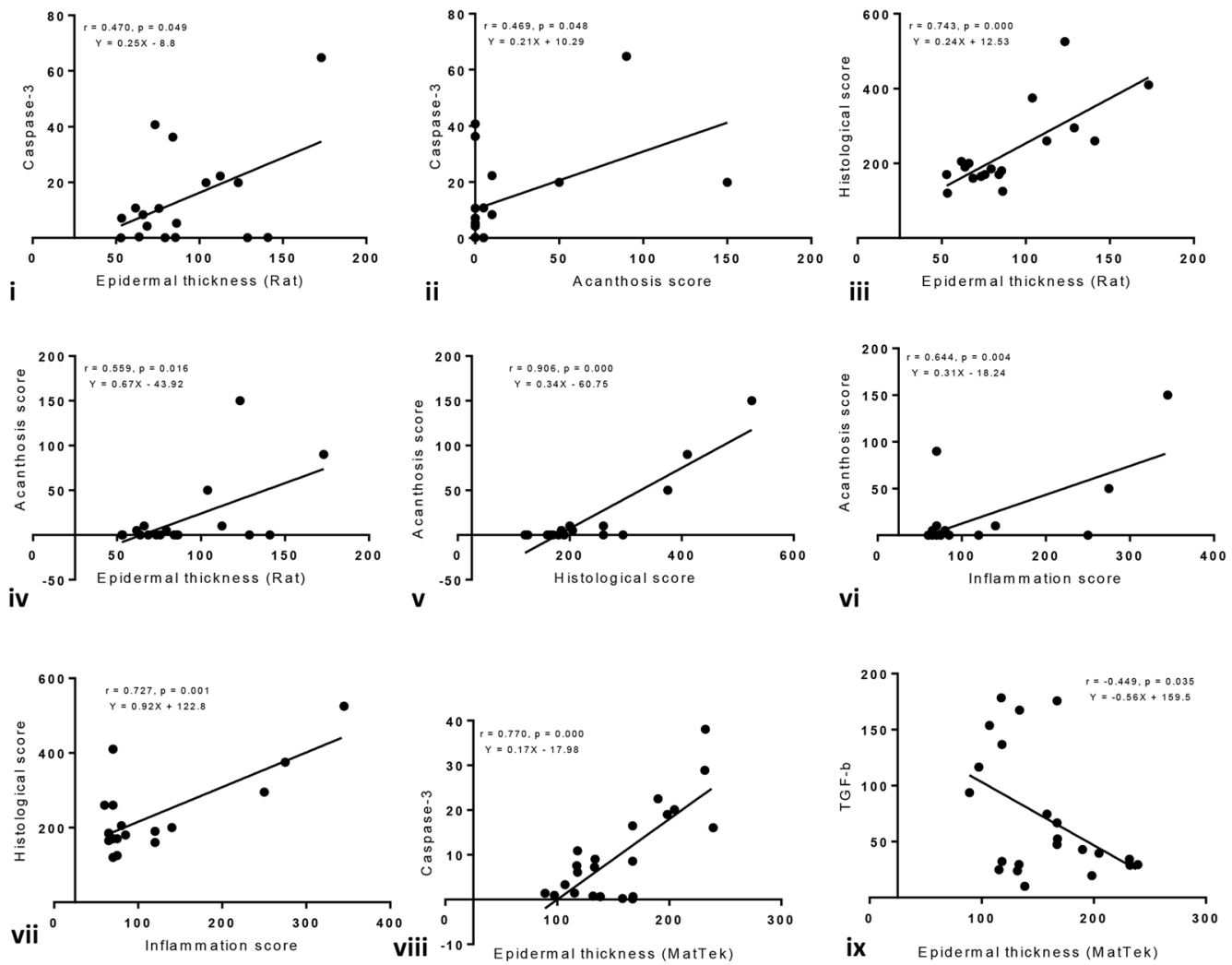

Figure 4. Epidermal psoriatic cytokine levels in artificial psoriatic human skin and imiquimod-induced psoriasis rat skin. (A) $\beta$-defensin (i-v) and CCL-20 (vi-x) immunolabeling of the rat psoriatic epidermis at day 7 post-treatment (magnification, $\times 400$ ). (i, vi) non-treatment; (ii, vii) $2.5 \%$ extract; (iii, viii) $5 \%$ extract; (iv, ix) betamethasone; (v, x) calcitriol (D3). Bar graph comparing the expression levels of $\beta$-defensin and CCL-20 among the various groups in the artificial psoriatic (xi) and rat psoriatic (xii) skins. (B) Graphs (i-ix) showing correlations among the expression levels of the markers and the histopathological scores. 
A
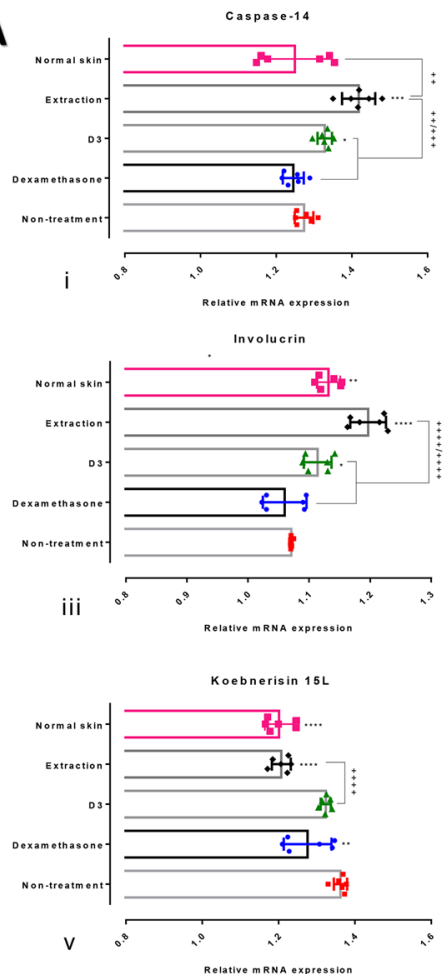

B
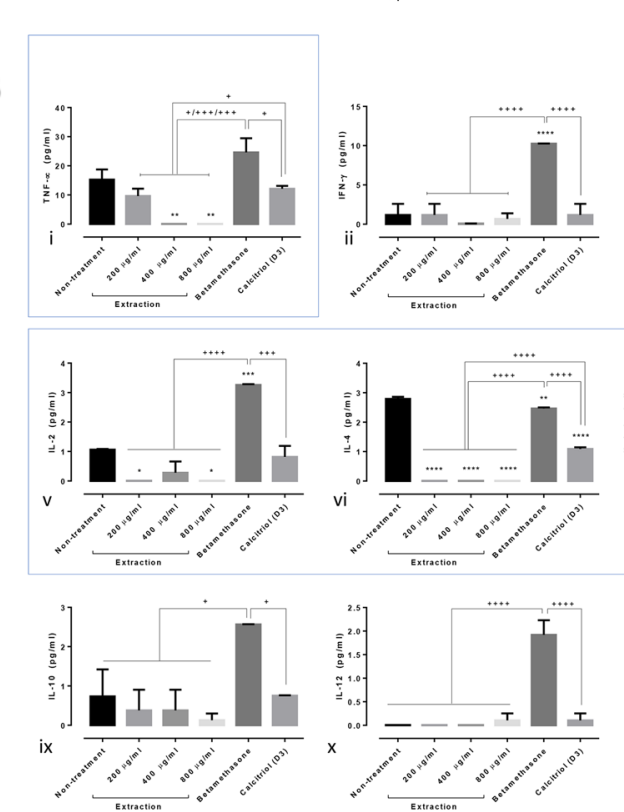

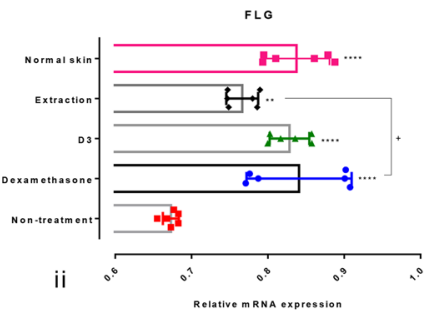

Psoriasin
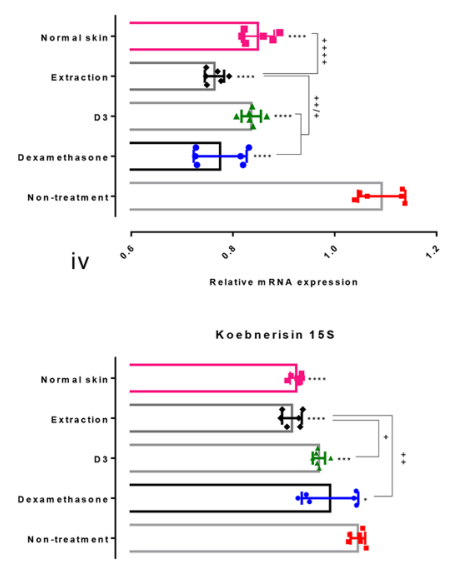

vi $x^{\circ} \quad n^{2}$
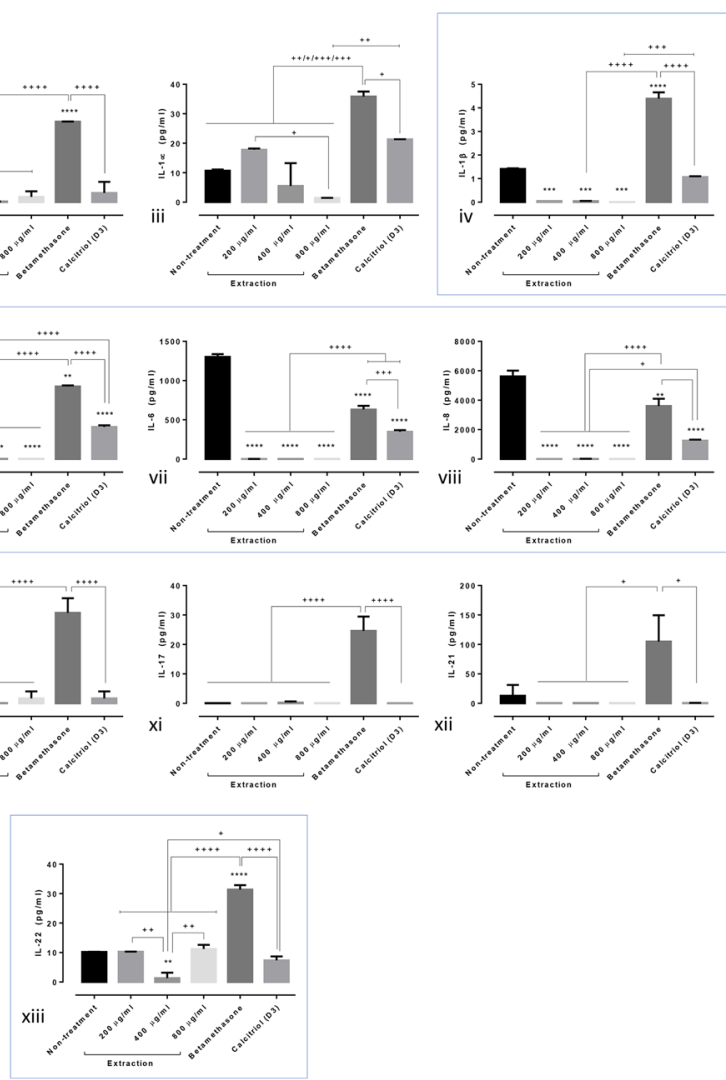

Figure 5. Gene expression and cytokine production levels in artificial psoriatic human skin. (A) Relative mRNA expression levels among the artificial psoriatic skin specimens with and without treatment. (i-vi) mRNA expression levels of caspase-14, filaggrin, involucrin, psoriasin, koebnerisin 15L and koebnerisin 15S, respectively. (B) Pro- and anti-inflammatory cytokines in the supernatants of the artificial psoriatic skins with or without treatment for 8 days. (i-xiii) Results of the Bio-Plex Multiplex immunoassay for tumour necrotic factor (TNF)- $\alpha$, interferon (IFN)- $\gamma$, interleukin (IL)-1 $\alpha$, IL-1 $\beta$, IL-2, IL-4, IL-6, IL-8, IL-10, IL-12, IL-17, IL-21 and IL-22. 


\begin{tabular}{|c|c|c|c|c|c|c|c|c|}
\hline \multirow{2}{*}{$\begin{array}{l}\text { Genes/ } \\
\text { markers }\end{array}$} & \multicolumn{8}{|c|}{ Correlation coefficient (p-value) } \\
\hline & Caspase-3 & Nrf-2 & IL-10 & TGF- $\beta$ & Involucrin & Koebnerisin15L & Koebnerisin15S & Psoriasin \\
\hline Caspase- 14 & & $0.702(0.000)$ & & & \begin{tabular}{|l}
0.854 \\
$(0.000)$
\end{tabular} & & & $\begin{array}{c}-0.667 \\
(0.001)\end{array}$ \\
\hline Involucrin & & $0.879(0.000)$ & $0.615(0.002)$ & $0.564(0.006)$ & & $-0.583(0.004)$ & $-0.467(0.028)$ & $\begin{array}{c}-0.641 \\
(0.001)\end{array}$ \\
\hline $\begin{array}{l}\text { Koebneris- } \\
\text { in15L }\end{array}$ & & $\begin{array}{l}-0.715 \\
(0.000)\end{array}$ & $\begin{array}{l}-0.861 \\
(0.000)\end{array}$ & $\begin{array}{c}-0.502 \\
(0.017)\end{array}$ & & & $0.667(0.001)$ & \\
\hline $\begin{array}{l}\text { Koebneri- } \\
\sin 15 \mathrm{~S}\end{array}$ & & $\begin{array}{c}-0.730 \\
(0.000)\end{array}$ & $\begin{array}{c}-0.640 \\
(0.001)\end{array}$ & & & & & $0.542(0.009)$ \\
\hline FLG & $\begin{array}{c}-0.592 \\
(0.004)\end{array}$ & & & & & & & \\
\hline Psoriasin & & $\begin{array}{l}-0.633 \\
(0.002)\end{array}$ & & & & & $0.542(0.005)$ & \\
\hline
\end{tabular}

Table 2. Significant correlations between the gene expression levels and some immunohistochemical markers.
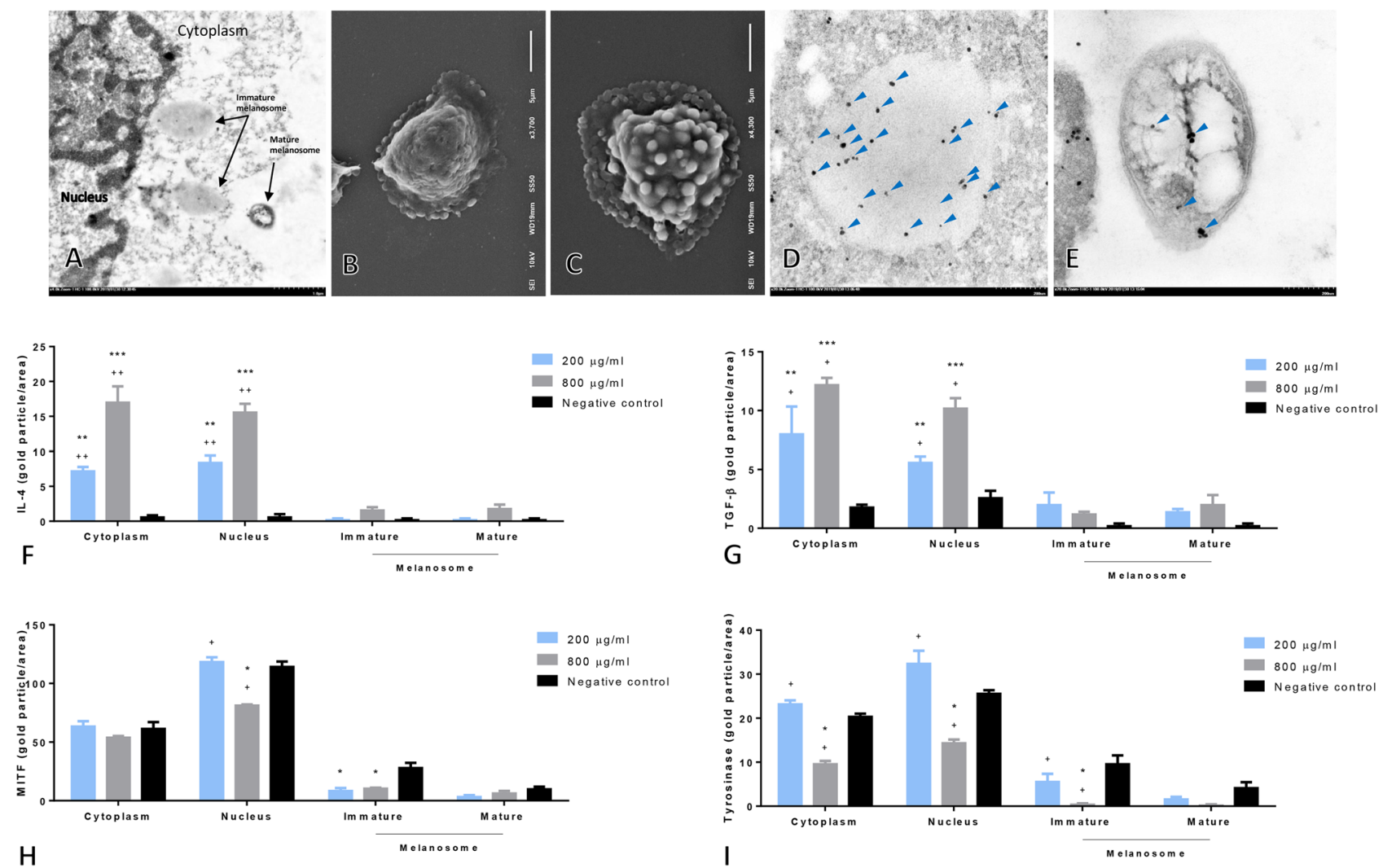

Figure 6. Antityrosinase, anti-melanocytic and anti-allergic properties of rice extract on melanocytes. Transmission electron image of allergic melanocyte (A) and scanning electron images of allergic melanocyte with (B) or without treatment using rice extract (C). Immunogold labelling of MITF in immature (D) and mature melanocytes (E). Bar graph comparisons of IL-4, TGF- $\beta$, MITF and tyrosinase expression levels in melanocytes among the various groups $(\mathbf{F}-\mathbf{I})$.

easily available and inexpensive, and it is known to exhibit low oral bioavailability; however, new delivery system formulations and clinical trials are being conducted to develop appropriate forms of this product. Cannabinoid extract containing tetrahydrocannabinol (THC) and cannabidiol (CBD) is used for the treatment of psoriasis. Both THC-mediated inhibition of keratinocyte proliferation and cannabinol (CB)-1 activation are believed to be crucial for the treatment of psoriasis via the reduction of inducible factor-1 a (HIF-1 $\alpha$ ), vascular endothelial growth factor, basic fibroblast growth factor (bFGF), angiopoietin-2, IL-8, IL-17 and IL-2 ${ }^{13}$. Anthocyanins demonstrate potent antioxidative, anti-inflammatory and anti-proliferative properties and are obtained from natural pigmented fruits, rice and vegetables ${ }^{20}$. Anthocyanin and its derivatives decrease psoriasis by inhibiting key kinases and cytokines ${ }^{15}$, reducing keratinocyte proliferation ${ }^{16,17}$ and controlling the expression of late cornified envelope genes ${ }^{14,21}$. 
New candidates for the treatment of psoriasis are being investigated. In the current study, the effect of crude extract from Oryza sativa L.: SRNC05053-6-2 consisting of high levels of anthocyanin were evaluated. The results from in vitro and in vivo studies indicated that the extract exhibited some beneficial effects on psoriasis, as demonstrated by the reduction in the epidermal pathologies (Fig. 1A, B). Epidermal thickening, acanthosis, hyperkeratosis and inflammation were reduced along with a decrease in the degree of cellular apoptosis (downregulation of caspase-3; Fig. 2A). The antioxidative environment as enhanced (upregulation of Nrf-2; Fig. 2B); furthermore, the expression levels of anti-inflammatory cytokines were increased (upregulations of IL-10 and TGF- $\beta$; Fig. 3) and those of some chemokines were decreased (downregulations of $\beta$-defensin and CCL-20; Fig. 4A). qRT-PCR indicated that compared with the standard treatments, the extract could maintain the homeostasis as well as the functional and structural integrities of the epidermis by upregulating the mRNA expression levels of caspase-14, involucrin and filaggrin, thereby improving the skin conditions (Fig. 5A). The depletion of anti-microbial peptides such as psoriasin and koebnerisin (15L and $15 \mathrm{~S}$ ), which are mainly found in inflamed psoriatic skin, leads to epidermal degeneration and necrosis ${ }^{22}$ and enhances the curable effect of the extract on psoriatic skin conditions. In the present study, the reduction in epidermal apoptosis and improvement in epidermal integrity were closely related to the antioxidative property of the extract via Nrf- 2 expression. This finding is consistent with that reported by Lee et al. ${ }^{23}$, wherein psoriasis vulgaris was found to be attenuated in human skin following a reduction in the redox burden and the subsequent oxidative damage to normal keratinocytes through the activation of the Nrf-2 pathway. In the present study, epidermal thickening was positively correlated with the expression levels of caspase-3, an apoptotic inducer, and negatively correlated with that of TGF- $\beta$, a multipotent cytokine that regulates both cell growth and differentiation. Owing to its anti-inflammatory and anti-keratinocyte proliferative activities, the downregulation of TGF- $\beta$ signalling was reported in human psoriatic conditions $^{24}$. In addition, the extract demonstrated antimelanogenic properties through its anti-inflammatory and antityrosinase activities, as characterised by the upregulation of IL- 4 and TGF- $\beta$ and downregulation of MITF and tyrosinase, respectively, in allergic melanocytes (Fig. 6), thus leading to a reduction in melanosome production (Fig. 6A-E). Owing to its antityrosinase property, anthocyanin extract can inhibit melanogenesis in the melanocyte ${ }^{25,26}$. This effect of the extract on melanin biogenesis is very important for improving psoriatic skin conditions during treatment with PUVA or photochemotherapy, which can lead to sub-epidermal melanincontaining phagocytosis ${ }^{27}$. Furthermore, researchers well recognised that melanocytes act as new co-operated target cells with keratinocytes in provoking autoantigens and autoimmunity in psoriasis ${ }^{2}$. Therefore, the beneficial effect of the extract on melanogenesis inhibition is desired for the development of anti-psoriactic agents, in addition to the anti-inflammatory and anti-epidermal thickening effects.

Several psoriasis-related cytokines and chemokines are considered important factors in the pathogenesis of psoriasis as mentioned above. In the present study, Bio-Plex Multiplex immunoassay revealed that the rice extract reduced inflammatory responses by (1) regulating the innate immune response via enhanced expression levels of TGF- $\beta$ and IL-10, (2) modulating the adaptive immune response through the downregulation of IL-6, IL-8, IL-22 and TNF- $\alpha$ and (3) attenuating the auto-amplification loop via the reduction of IL-20, CCL-20 and $\beta$-defensin. Cumulatively, these findings demonstrate that the beneficial effects of the extract in ameliorating the severity of psoriasis.

In summary, the present study explored the antipsoriatic property of the crude extract from Oryza sativa $\mathrm{L}$.: SRNC05053-6-2 or Mali-Nil-Surin rice using in vitro and in vivo models. The extract improved epidermal integrity by maintaining the expression levels of psoriasin, $\beta$-defensin, koebnerisin $15 \mathrm{~L}$, koebnerisin 15S, caspase-14, involucrin and filaggrin. In addition, inflammation had decreased owing to the antioxidative effects exerted via Nrf- 2 and the immunomodulatory effects exerted by the regulation of IL-10, TGF- $\beta$, IL-6, IL-8, IL-20, IL-22, TNF- $\alpha$ and CCL-20 levels. Therefore, $O$ sativa L.: SRNC05053-6-2 extract appears to be a potential candidate for the treatment of psoriasis.

\section{Methods}

Ethics statement. The animal experiments were performed with the permission of the National Laboratory Animal Center-ACUC, Mahidol University (approval number RA2019-21). The Thai Animals for Scientific Purposes Act, B.E. 2558, and the Guidelines for the use of animals of the National Research Council of Thailand were implemented. Sprague-Dawley rats were obtained from the National Laboratory Animal Center at Mahidol University. They were housed in a temperature-, ventilation- and humidity-controlled room under 12-h/12-h light/dark cycle conditions and fed with a standard diet ad libitum.

Extract preparation. Mali-Nil-Surin rice (Oryza sativa L.: SRNC05053-6-2) was obtained from organic farms certified by the Ministry of Agricultural and Cooperative, Thailand. The pericarp was milled and rice extract was prepared via ethanolic extraction by the Thai-China Flavours and Fragrances Industry Co., Ltd. (TCFF) using a hi-speed herb extractor (HX-50, UK). The main active ingredients of the extract (anthocyanin, ascorbic acid and $\alpha$-tocopherol) and its antioxidative properties [total antioxidative activity; ferric reducing antioxidative power (FRAP) and 1,1-diphenyl-2-picrylhydrazyl (DPPH) assays] were evaluated by TCFF and the Nutrition Institute, Mahidol University, Thailand.

In vitro and in vivo effects of the extract on psoriasis. In vitro psoriasis model. Full-thickness threedimensional reconstituted human skin models of psoriasis (PSE) and normal skin (NSE; MatTek, USA) were used to evaluate the ability of the extract to alleviate psoriasis. The PSE consisted of human psoriatic keratinocytes, highly differentiated epidermis and collagen-contracted fibroblasts, whereas the NSE consisted of intact human keratinocytes and other cells. A full-thickness artificial skin specimen was equilibrated at $37^{\circ} \mathrm{C}$ and $5 \% \mathrm{CO}_{2}$ for $24 \mathrm{~h}$ when received and maintained in SOR-300-FT-MM media throughout the experiment. Pre- 


\begin{tabular}{|c|c|c|}
\hline Gene & Forward & Reverse \\
\hline FLG & 5'-AAGGTTCACATTTATTGCCAAA-3' & 5'-GGATTTGCCGAAATTCCTTT-3' \\
\hline$\beta$-Actin & $\begin{array}{l}\text { 5'-TGACGGGGTCACCCACACTGTGCCCAT } \\
\text { CTA-3' }\end{array}$ & $\begin{array}{l}\text { 5'-CTAGAAGCATTTGCGGTGGACGATGGA } \\
\text { GGG-3' }\end{array}$ \\
\hline Caspase-14 & 5'-AAATGAGCAATCCGCGGTCTTTGG-3' & 5'-CCGTGGAATAAACGTGCAAGGCAT-3' \\
\hline Involucrin & 5'-CTCCACCAAAGCCTCTGC-3' & 5'-CTGCTTAAGCTGCT GCTC-3' \\
\hline TGase-1 & 5'-TGAATAGTGACAAGGTGTACTGGCA-3' & 5'-GTGGCCTGAGACATTGAGCAGCAT-3' \\
\hline psoriasin-hS100A7 & 5'-AGACGTGATGACAAGATTGAC-3' & 5'-TGTCCTTTTTCTCAAAGACGTC-3' \\
\hline Koebnerisin 15S-hS100A15S & 5'-CAAGTTCCTTCTGCTCCATCTTAG-3' & 5'-AGCCTTCAGGAAATAAAGACAATC-3' \\
\hline Koebnerisin 15L-hS100A15L & 5'-ACGTCACTCCTGTCTCTCTTTGCT-3' & 5'-TGATGAATCAACCCATTTCCTGGG-3' \\
\hline
\end{tabular}

Table 3. Primers used for qRT-PCR analysis.

warmed media with or without treatment $(200 \mu \mathrm{g} / \mathrm{ml}$ extract, $1 \mu \mathrm{g} / \mathrm{g}$ calcitriol with $0.01 \mu \mathrm{g} / \mathrm{g}$ betamethasone [Xamiol] and $0.4 \mathrm{mg} / \mathrm{ml}$ dexamethasone) was added to the artificial skin and changed every 2 days until 8 days post-treatment. The experiments were performed in triplicates. The supernatant was collected at $-80{ }^{\circ} \mathrm{C}$ to determine the concentrations of the cytokines. The artificial skin was divided into two parts: one was fixed in $10 \%$ neutral buffer formalin (NBF) for histopathological examination, and the other was frozen at $-80{ }^{\circ} \mathrm{C}$ to evaluate the expression levels of psoriasis-related genes using qRT-PCR.

Peptidoglycan (PEG) induced melanocyte allergy. Recently, researchers showed that in addition to keratinocytes, melanocytes contribute to the severity of psoriasis by targeting the human leukocyte antigen class I allele, the main psoriasis risk gene ${ }^{2}$. To demonstrate the anti-inflammatory and antityrosinase activities of the extract, a melanocyte allergic model was created via PEG-induced inflammation ${ }^{28}$. The human melanoma cell line (ATCC CRL-1676) was cultured in Eagle's Minimum Essential Medium supplemented with $10 \%$ (v/v) heat-inactivated fetal bovine serum and $1 \%(\mathrm{v} / \mathrm{v})$ penicillin/streptomycin. The cells were cultured (density, $10^{6}$ cells $\left./ \mathrm{ml}\right)$ and maintained at $37^{\circ} \mathrm{C}$ with $5 \% \mathrm{CO}_{2}$ in a humidified incubator. The melanocytes were co-cultured with $10 \mu \mathrm{g} / \mathrm{ml}$ of $S$. aureus PEG (Sigma-Aldrich, USA) for $24 \mathrm{~h}$. After induction, the cells were treated with or without 200 and $800 \mu \mathrm{g} / \mathrm{ml}$ of the rice extract for $24 \mathrm{~h}$. After the cells were trypsinised with $0.25 \%$ Trypsin-EDTA and washed in sucrose phosphate buffer (SPB), they were centrifuged at $3500 \mathrm{rpm}\left(4^{\circ} \mathrm{C}\right)$ for $5 \mathrm{~min}$. The melanocyte pellets were collected and fixed in $2.5 \%$ glutaraldehyde and SPB for $1 \mathrm{~h}$ at room temperature.

Induced psoriasis rat model. To induce psoriasis in the rat model, $62.5 \mathrm{mg}$ of imiquimod was topically applied to the dorsal shaved rat skin (limited to $3 \times 3 \mathrm{~cm}^{2}$ area) daily for 7 days ${ }^{29}$. Thirty rats were equally divided into six groups ( 5 rats/group) based on the following different test materials used for 7 days: cream base (non-treatment group); $2.5 \%, 5 \%$ and $10 \%$ of rice extract; $3 \mu \mathrm{g} / \mathrm{ml}$ of calcitriol ointment; and $0.1 \%$ betamethasone. After the induction period, imiquimod was continually applied to the rats to maintain the psoriatic skin lesion. All test materials were applied 2 times/ day (b.i.d.) after the application of imiquimod for $1 \mathrm{~h}$. The skin lesion was grossly scored every day by focusing on the erythema, scale and thickening using four grades: $0=$ absent, $1=$ mild, $2=$ moderate and $3=$ severe. At the end of the study, all rats were humanely euthanised by an overdose of carbondioxide inhalation. The skin lesions were collected and preserved in 10\% NBF for histopathological examination.

qRT-PCR. RNA from artificial skin was isolated using TRIzol Reagent (Invitrogen, USA), according to the manufacturer's instructions. Complementary DNA synthesis and amplification were performed using the CFX96 Touch Real-time PCR Detection system thermocycler and KAPA SYBR FAST One-Step qRT-PCR Kit (KAPABIOSYSTEMS, USA), according to the manufacturers' protocols. The primer pairs used are presented in Table 3. The level of mRNA was normalised to the levels of $\beta$-actin or TGase-1 mRNA. Subsequently, the relative mRNA expression levels were calculated using the $2^{-\Delta \Delta C t}$ method.

Bio-plex multiplex immunoassay. To determine the cytokines involved in the innate and adaptive immune responses and the auto-amplification loop immunity, which are closely related to the pathogenesis of psoriasis, the Bio-Plex Multiplex immunoassay (BioRad, USA) was performed. Luminex magnetic beads were used to quantify some of the following cytokines: tumour necrotic factor (TNF)- $\alpha$, interferon (IFN)- $\gamma$, interleukin (IL)-1 $\alpha$, IL-1 $\beta$, IL-2, IL-4, IL-6, IL-8, IL-10, IL-12, IL-17, IL-21 and IL-22. After harvesting the cells from the artificial skin following treatment for 7 days, the supernatant was thawed and centrifuged at $1000 \mathrm{~g}$ for $10 \mathrm{~min}$. The negative and positive controls were supplied in the kit.

Histopathological examination. To assess the severity of the histopathological changes in the artificial and rat psoriatic skins, standard tissue processing consisting of dehydration, infiltration, embedding, sectioning and staining with hematoxylin and eosin was performed. The histopathological score was ascertained following examination under a light microscope. The epidermal thicknesses of the artificial and rat psoriatic skin specimens were measured using an imaging analysis programme (ImageJ Version 1.36; National Institutes of Health; Bethesda, MD, USA). The histopathological changes in rat psoriatic skin were determined by observing the presence of acanthosis and elongation of rete ridges, the thickness of the epidermis and the presence of hyper- 
keratosis, folliculitis and dermatitis; the changes were scored using the $\mathrm{H}$-score, as described previously ${ }^{30-39}$. The $\mathrm{H}$-score $(0-300)$ was calculated by multiplying the severity score $(0-3 ; 0=$ absent, $1=$ mild, $2=$ moderate and $3=$ severe) with the extent of distribution $(0-100 \%)$.

Immunohistochemical studies. To demonstrate the resolutive, antioxidative and immunomodulatory properties of the extract on both artificial and rat psoriasis skin specimens, the immunostaining, as described in our recent studies ${ }^{30-35,38,39}$, of caspase-3, nuclear factor erythroid 2-related factor (Nrf)-2, interleukin (IL)10 , TGF- $\beta, \beta$-defensin and CCL-20 were performed using rabbit isotype polyclonal antibodies (MyBioSource, USA). After deparaffinisation and dehydration, the antigenicity of the section was enhanced using heated citrate buffer ( $\mathrm{pH}$ 6.0). The blocking steps were performed using peroxidase, and non-specific binding was blocked using EnVision FLEX/ horseradish peroxidase (HRP) blocking reagent (DAKO, Denmark, K8002). The sections were incubated with the primary antibody for $1 \mathrm{~h}$ and labelled with polymer HRP anti-mouse/rabbit (DAKO) for $20 \mathrm{~min}$, followed by visualisation with diaminobenzidine (DAB; DAKO). Finally, the sections were counterstained with hematoxylin and mounted with DEPEX (Electron Microscopy Sciences, USA). The immunolabeling of all the markers of interest was semi-quantified based on the $\mathrm{H}$-score (percentage of expression $\times$ intensity score) using the image analysis programme to localise an area of expression. Briefly, psoriatic full-thickness skin lesions were captured as colour images (at least five images/ animal). The immunolabeled area was located using the threshold mode, and the images were converted to grayscale. Finally, the area of expression (percentage) was measured. The intensity score was graded as follows: $0=$ negative staining; $1=$ low-intensity staining; $2=$ moderate-intensity staining; and 3 = high-intensity staining.

Electron microscopy. Transmission electron microscopy. All the fixed melanocyte pellets were fixed with $1 \%$ osmium tetroxide in $0.1 \mathrm{M} \mathrm{SPB}$ for $1 \mathrm{~h}$ at room temperature. Next, the specimens were dehydrated in graded ethanol, infiltrated with LR White resin (EMS, USA), embedded in capsule beams and finally polymerised at $65^{\circ} \mathrm{C}$ for $48 \mathrm{~h}$. All specimens were cut into $90-100-\mathrm{nm}$ thick section and placed on a nickel grid for immunogold staining.

Scanning electron microscopy. Glutaraldehyde-fixed melanocytes were washed with $0.1 \mathrm{M}$ SPB and secondarily fixed with $1 \%$ osmium tetroxide in $0.1 \mathrm{M} \mathrm{SPB}$ for $1 \mathrm{~h}$ at room temperature. The pellets were dehydrated with graded ethyl-alcohol, smeared on a glass coverslip, dried in a desiccator and coated with gold film up to a thickness of $20 \mathrm{~nm}$ using a sputter coater (K550; EMITECH, UK). All specimens were examined under a scanning electron microscope (JSM-6610LV; JEOL, Japan) with 10-kV acceleration voltage to determine the density of the melanosome in the melanocyte.

Immunogold labelling. To examine the anti-inflammatory and antimelanogenic capacities of the extract on PEG-induced melanocyte inflammation, immunogold electron microscopy was performed, as described in our recent studies ${ }^{32-34}$, using rabbit anti-IL-4, TGF- $\beta$, MITF and tyrosinase (MyBioSource, USA). Sections were blocked using $50 \mathrm{mM}$ glycine and 5\% bovine serum albumin (BSA; EMS, USA). The sections were incubated with primary antibodies for $1 \mathrm{~h}$ and then with immunoglobulin (Ig)G conjugated with $10 \mathrm{~nm}$ gold particles (EMS, USA) for $1 \mathrm{~h}$. A silver enhancement kit (Aurion R-Gent SE-EM kit, EMS, USA) was used after rigorously washing the sections with distilled water. Finally, the sections were stained with lead citrate and uranyl acetate and examined under a transmission electron microscope (HT7700, HITACHI, Japan). The number of gold particles labelled in the nucleus and cytoplasm of the mature and immature melanosome was counted (in at least 50 cells/group).

Statistics. Statistical analysis was performed using GraphPad Prism version 5, and data are expressed as mean \pm standard error of the mean. Statistical significance was denoted as $p<0.05\left({ }^{*},{ }^{* *},{ }^{* *}\right.$, or ${ }^{* * * *}$ referred to a significant difference to non-treated group at $p$-value $<0.05,0.01,0.001$, or 0.0001 , respectively:,+++ , +++ or ++++ referred to a significant difference between match pair at $p$-value $<0.05,0.01,0.001$ or 0.0001 , respectively). The Kolmogorov-Smirnov test was used to determine the data distribution, and appropriate parametric and non-parametric $t$-tests or analysis of variance were chosen to compare the differences in histopathological, ultrastructural and immunohistochemical changes among groups. Spearman's correlation test was used to determine the correlations in this study.

Data availability. The data sets used and/or analysed during the current study are available from the corresponding author upon request.

Received: 12 July 2020; Accepted: 5 October 2020

Published online: 19 October 2020

\section{References}

1. Abdel-Naser, M. B. et al. Increased activity and number of epidermal melanocytes in lesional psoriatic skin. Dermatology 232, 425-430. https://doi.org/10.1159/000447535 (2016).

2. Arakawa, A. et al. Melanocyte antigen triggers autoimmunity in human psoriasis. J. Exp. Med. 212, 2203-2212. https://doi. org/10.1084/jem.20151093 (2015).

3. Krueger, J. G. An autoimmune "attack" on melanocytes triggers psoriasis and cellular hyperplasia. J. Exp. Med. 212, 2186. https:// doi.org/10.1084/jem.21213insight3 (2015). 
4. Prinz, J. C. Melanocytes: target cells of an HLA-C ${ }^{\star} 06: 02$-restricted autoimmune response in psoriasis. J. Investig. Dermatol 137, 2053-2058. https://doi.org/10.1016/j.jid.2017.05.023 (2017).

5. Strassner, J. P., Rashighi, M. \& Harris, J. E. Melanocytes in psoriasis: convicted culprit or bullied bystander?. Pigment Cell Melanoma Res. 29, 261-263. https://doi.org/10.1111/pcmr.12470 (2016).

6. Ferreira, B. I., Abreu, J. L., Reis, J. P. \& Figueiredo, A. M. Psoriasis and associated psychiatric disorders: a systematic review on etiopathogenesis and clinical correlation. J. Clin. Aesthet. Dermatol. 9, 36-43 (2016).

7. Monteleone, G., Pallone, F., MacDonald, T. T., Chimenti, S. \& Costanzo, A. Psoriasis: from pathogenesis to novel therapeutic approaches. Clin. Sci. (London) 120, 1-11. https://doi.org/10.1042/CS20100163 (2011).

8. Surin, S., Surayot, U., Seesuriyachan, P., You, S. \& Phimolsiripol, Y. Antioxidant and immunomodulatory activities of sulphated polysaccharides from purple glutinous rice bran (Oryza sativa L.). Int. J. Food Sci. Technol. 53, 994-1004 (2018).

9. Thiranusornkij, L., Thamnarathip, P., Chandrachai, A., Kuakpetoon, D. \& Adisakwattana, S. Physicochemical properties of Hom Nil (Oryza sativa) rice flour as gluten free ingredient in bread. Foods 7, 1-13. https://doi.org/10.3390/foods7100159 (2018).

10. Saiyot, A. et al. Mali Nil Surin (Mali Dam 2), a non glutinous rice variety. Thai Rice Res. J. 8, 8-25 (2017).

11. Vollono, L. et al. Potential of curcumin in skin disorders. Nutrients 11, 1-25. https://doi.org/10.3390/nu11092169 (2019).

12. Yousef, S. A. et al. Mechanistic evaluation of enhanced curcumin delivery through human skin in vitro from optimised nanoemulsion formulations fabricated with different penetration enhancers. Pharmaceutics 11, 1-20. https://doi.org/10.3390/pharmaceut ics11120639 (2019).

13. Sheriff, T., Lin, M. J., Dubin, D. \& Khorasani, H. The potential role of cannabinoids in dermatology. J. Dermatol. Treat. 30, 1-7. https://doi.org/10.1080/09546634.2019.1675854 (2019).

14. Austin, H. R. et al. Regulation of late cornified envelope genes relevant to psoriasis risk by plant-derived cyanidin. Biochem. Biophys. Res. Commun. 443, 1275-1279. https://doi.org/10.1016/j.bbrc.2013.12.128 (2014).

15. Chamcheu, J. C. et al. Dual inhibition of PI3K/Akt and mTOR by the dietary antioxidant, delphinidin, ameliorates psoriatic features in vitro and in an imiquimod-induced psoriasis-like disease in mice. Antioxid. Redox Signal 26, 49-69. https://doi.org/10.1089/ ars.2016.6769 (2017).

16. Chamcheu, J. C. et al. Delphinidin, a dietary antioxidant, induces human epidermal keratinocyte differentiation but not apoptosis: studies in submerged and three-dimensional epidermal equivalent models. Exp. Dermatol. 22, 342-348. https://doi.org/10.1111/ exd.12140 (2013).

17. Chamcheu, J. C. et al. Prodifferentiation, anti-inflammatory and antiproliferative effects of delphinidin, a dietary anthocyanidin, in a full-thickness three-dimensional reconstituted human skin model of psoriasis. Skin Pharmacol. Physiol. 28, 177-188. https:// doi.org/10.1159/000368445 (2015)

18. Han, S. J. et al. Metabolism of cyanidin-3-O-beta-D-glucoside isolated from black colored rice and its antiscratching behavioral effect in mice. J. Food Sci. 74, H253-258. https://doi.org/10.1111/j.1750-3841.2009.01327.x (2009).

19. Pal, H. C. et al. Topical application of delphinidin reduces psoriasiform lesions in the flaky skin mouse model by inducing epidermal differentiation and inhibiting inflammation. Br. J. Dermatol. 172, 354-364. https://doi.org/10.1111/bjd.13513 (2015).

20. Westfall, A., Sigurdson, G. T., Rodriguez-Saona, L. E. \& Giusti, M. M. Ex Vivo and in vivo assessment of the penetration of topically applied anthocyanins utilizing ATR-FTIR/PLS regression models and HPLC-PDA-MS. Antioxidants (Basel) 9, 1-14. https://doi. org/10.3390/antiox9060486 (2020).

21. Hoss, E. et al. Control of late cornified envelope genes relevant to psoriasis risk: upregulation by 1,25-dihydroxyvitamin D3 and plant-derived delphinidin. Arch. Dermatol. Res. 305, 867-878. https://doi.org/10.1007/s00403-013-1390-1 (2013).

22. Wolf, R., Ruzicka, T. \& Yuspa, S. H. Novel S100A7 (psoriasin)/S100A15 (koebnerisin) subfamily: highly homologous but distinct in regulation and function. Amino Acids 41, 789-796. https://doi.org/10.1007/s00726-010-0666-4 (2011).

23. Lee, Y. J. et al. Pro-oxidant status and Nrf2 levels in psoriasis vulgaris skin tissues and dimethyl fumarate-treated HaCaT cells. Arch. Pharm. Res. 40, 1105-1116. https://doi.org/10.1007/s12272-017-0955-5 (2017).

24. Han, G. et al. A role for TGFbeta signaling in the pathogenesis of psoriasis. J. Investig. Dermatol. 130, 371-377. https://doi. org/10.1038/jid.2009.252 (2010).

25. Hwang, J. M., Kuo, H. C., Lin, C. T. \& Kao, E. S. Inhibitory effect of liposome-encapsulated anthocyanin on melanogenesis in human melanocytes. Pharm. Biol. 51, 941-947. https://doi.org/10.3109/13880209.2013.771376 (2013).

26. Westfall, A., Sigurdson, G. T. \& Giusti, M. M. Antioxidant, UV protection, and antiphotoaging properties of anthocyanin-pigmented lipstick formulations. J. Cosmet. Sci. 70, 63-76 (2019).

27. Stuttgen, G., Brinkmann-Raestrupp, I., Haller, L., Kentsch, V. \& Schalla, W. Melanin granula distribution and phagocytosis in psoriasis vulgaris after PUVA therapy. Arch. Dermatol. Res. 264, 29-35. https://doi.org/10.1007/BF00417276 (1979).

28. Aramwit, P., Luplertlop, N., Kanjanapruthipong, T. \& Ampawong, S. Effect of urea-extracted sericin on melanogenesis: potential applications in post-inflammatory hyperpigmentation. Biol. Res. 51, 54. https://doi.org/10.1186/s40659-018-0204-5 (2018).

29. Lin, Y. K., Yang, S. H., Chen, C. C., Kao, H. C. \& Fang, J. Y. Using imiquimod-induced psoriasis-like skin as a model to measure the skin penetration of anti-psoriatic drugs. PLoS ONE 10, e0137890. https://doi.org/10.1371/journal.pone.0137890 (2015).

30. Ampawong, S. \& Aramwit, P. Tolerogenic responses of CD206+, CD83+, FOXP3+, and CTLA-4 to sericin/polyvinyl alcohol/ glycerin scaffolds relevant to IL-33 and HSP60 activity. Histol. Histopathol. 31, 1011-1027. https://doi.org/10.14670/HH-11-733 (2016).

31. Ampawong, S. et al. A potential role for interleukin-33 and gamma-epithelium sodium channel in the pathogenesis of human malaria associated lung injury. Malar. J. 14, 389. https://doi.org/10.1186/s12936-015-0922-x (2015).

32. Ampawong, S., Isarangkul, D. \& Aramwit, P. Sericin improves heart and liver mitochondrial architecture in hypercholesterolaemic rats and maintains pancreatic and adrenal cell biosynthesis. Exp Cell Res 358, 301-314. https://doi.org/10.1016/j.yexcr.2017.07.001 (2017).

33. Ampawong, S., Isarangkul, D. \& Aramwit, P. Sericin ameliorated dysmorphic mitochondria in high-cholesterol diet/streptozotocin rat by antioxidative property. Exp Biol Med (Maywood) 242, 411-421. https://doi.org/10.1177/1535370216681553 (2017).

34. Ampawong, S., Isarangkul, D., Reamtong, O. \& Aramwit, P. Adaptive effect of sericin on hepatic mitochondrial conformation through its regulation of apoptosis, autophagy and energy maintenance: a proteomics approach. Sci Rep 8, 14943. https://doi. org/10.1038/s41598-018-33372-4 (2018).

35. Ampawong, S. \& Luplertlop, N. Experimental scedosporiosis induces cerebral oedema associated with abscess regarding Aquaporin-4 and Nrf-2 depletions. Biomed Res Int 2019, 6076571. https://doi.org/10.1155/2019/6076571 (2019).

36. Kengkoom, K. et al. Effects on high cholesterol-fed to liver, retina, hippocampus, and Harderian gland in Goto-Kakizaki rat. Int. J. Clin. Exp. Pathol. 6, 639-649 (2013).

37. Kengkoom, K., Tirawanchai, N. N., Angkhasirisap, W. \& Ampawong, S. Omeprazole preserves the RER in chief cells and enhances re-epithelialization of parietal cells with SOD and AQP-4 up-regulation in ethanol-induced gastritis rats. Exp. Ther. Med. 14, 5871-5880. https://doi.org/10.3892/etm.2017.5270 (2017).

38. Maknitikul, S., Luplertlop, N., Chaisri, U., Maneerat, Y. \& Ampawong, S. Featured article: immunomodulatory effect of hemozoin on pneumocyte apoptosis via CARD9 pathway, a possibly retarding pulmonary resolution. Exp. Biol. Med. (Maywood) 243, 395-407. https://doi.org/10.1177/1535370218757458 (2018).

39. Maknitikul, S., Luplertlop, N., Grau, G. E. R. \& Ampawong, S. Dysregulation of pulmonary endothelial protein C receptor and thrombomodulin in severe falciparum malaria-associated ARDS relevant to hemozoin. PLoS ONE 12, e0181674. https://doi. org/10.1371/journal.pone.0181674 (2017). 


\section{Acknowledgements}

This study was supported by the Faculty of Tropical Medicine, Mahidol University, and Agricultural Research Development Agency (Public Organization) under the project 'Efficacy study of Mali-Nil-Surin rice extraction to develop medicinal products for psoriasis alleviation; No. CRP6205030090'.

\section{Author contributions}

S.A. drafted the manuscript. All listed authors have contributed to read and approved the final manuscript for this submission. S.A., K.K., P.A. and T.B. conducted the animal experiments; S.A., P.S., P.A., W.M. and T.K. conducted in vitro experimentation, Bio-Plex Multiplex immunoassay and qRT-PCR; and S.A. and T.K. conducted histopathological and immunohistochemical studies.

\section{Competing interests}

The authors declare no competing interests.

\section{Additional information}

Correspondence and requests for materials should be addressed to S.A.

Reprints and permissions information is available at www.nature.com/reprints.

Publisher's note Springer Nature remains neutral with regard to jurisdictional claims in published maps and institutional affiliations.

(c) (i) Open Access This article is licensed under a Creative Commons Attribution 4.0 International cc) License, which permits use, sharing, adaptation, distribution and reproduction in any medium or format, as long as you give appropriate credit to the original author(s) and the source, provide a link to the Creative Commons licence, and indicate if changes were made. The images or other third party material in this article are included in the article's Creative Commons licence, unless indicated otherwise in a credit line to the material. If material is not included in the article's Creative Commons licence and your intended use is not permitted by statutory regulation or exceeds the permitted use, you will need to obtain permission directly from the copyright holder. To view a copy of this licence, visit http://creativecommons.org/licenses/by/4.0/.

(C) The Author(s) 2020 